\title{
Sharp maximal function inequalities and boundedness for commutators related to generalized fractional singular integral operators
}

\author{
Guangze Gu* and Mingjie Cai
}

"Correspondence: toggz@163.com College of Mathematics and Econometrics, Hunan University, Changsha, 410082, P.R. China

\begin{abstract}
In this paper, some sharp maximal function inequalities for the commutators related to certain generalized fractional singular integral operators are proved. As an application, we obtain the boundedness of the commutators on Lebesgue, Morrey and Triebel-Lizorkin spaces.

MSC: 42B20; 42B25
\end{abstract}

Keywords: singular integral operator; commutator; sharp maximal function; Morrey space; Triebel-Lizorkin space; $B M O$; Lipschitz function

\section{Introduction and preliminaries}

Let $b \in B M O\left(R^{n}\right)$ and $T$ be the Calderón-Zygmund singular integral operator. The commutator $[b, T]$ generated by $b$ and $T$ is defined by

$$
[b, T] f(x)=b(x) T f(x)-T(b f)(x) .
$$

By using a classical result of Coifman et al. (see [1]), we know that the commutator $[b, T]$ is bounded on $L^{p}\left(R^{n}\right)(1<p<\infty)$. Now, as the development of singular integral operators and their commutators, some new integral operators and their commutators are studied (see [2-7]). In $[5,8,9]$, the boundedness for the commutators generated by the singular integral operators and Lipschitz functions on Triebel-Lizorkin and $L^{p}\left(R^{n}\right)(1<p<\infty)$ spaces are obtained. In [10], some singular integral operators with general kernel are introduced, and the boundedness for the operators and their commutators generated by $B M O$ and Lipschitz functions are obtained (see $[10,11])$. Motivated by these, in this paper, we will prove some sharp maximal function inequalities for the commutator associated with certain generalized fractional singular integral operators and the $B M O$ and Lipschitz functions. As an application, we obtain the boundedness of the commutator on Lebesgue, Morrey and Triebel-Lizorkin space.

First, let us introduce some preliminaries. Throughout this paper, $Q$ will denote a cube of $R^{n}$ with sides parallel to the axes. For any locally integrable function $f$, the sharp maximal

\section{Springer}

(02014 Gu and Cai; licensee Springer. This is an Open Access article distributed under the terms of the Creative Commons Attribution License (http://creativecommons.org/licenses/by/2.0), which permits unrestricted use, distribution, and reproduction in any medium, provided the original work is properly cited. 
function of $f$ is defined by

$$
M^{\#}(f)(x)=\sup _{Q \ni x} \frac{1}{|Q|} \int_{Q}\left|f(y)-f_{Q}\right| d y,
$$

and here, and in what follows, $f_{Q}=|Q|^{-1} \int_{Q} f(x) d x$. It is well known that (see $\left.[12,13]\right)$

$$
M^{\#}(f)(x) \approx \sup _{Q \ni x} \inf _{c \in C} \frac{1}{|Q|} \int_{Q}|f(y)-c| d y .
$$

We say that $f$ belongs to $B M O\left(R^{n}\right)$ if $M^{\#}(f)$ belongs to $L^{\infty}\left(R^{n}\right)$ and define $\|f\|_{B M O}=$ $\left\|M^{\#}(f)\right\|_{L^{\infty}}$. It is well known that (see [7])

$$
\left\|f-f_{2^{k} Q}\right\|_{B M O} \leq C k\|f\|_{B M O} .
$$

Let

$$
M(f)(x)=\sup _{Q \ni x} \frac{1}{|Q|} \int_{Q}|f(y)| d y .
$$

For $\eta>0$, let $M_{\eta}(f)(x)=M\left(|f|^{\eta}\right)^{1 / \eta}(x)$.

For $0<\eta<n$ and $1 \leq r<\infty$, set

$$
M_{\eta, r}(f)(x)=\sup _{Q \ni x}\left(\frac{1}{|Q|^{1-r \eta / n}} \int_{Q}|f(y)|^{r} d y\right)^{1 / r} .
$$

The $A_{p}$ weight is defined by (see [12])

$$
\begin{aligned}
A_{p}= & \left\{w \in L_{\mathrm{loc}}^{1}\left(R^{n}\right): \sup _{Q}\left(\frac{1}{|Q|} \int_{Q} w(x) d x\right)\left(\frac{1}{|Q|} \int_{Q} w(x)^{-1 /(p-1)} d x\right)^{p-1}<\infty\right\}, \\
& 1<p<\infty,
\end{aligned}
$$

and

$$
A_{1}=\left\{w \in L_{\mathrm{loc}}^{p}\left(R^{n}\right): M(w)(x) \leq C w(x), \text { a.e. }\right\} .
$$

For $\beta>0$ and $p>1$, let $\dot{F}_{p}^{\beta, \infty}\left(R^{n}\right)$ be the homogeneous Triebel-Lizorkin space (see [9]). For $\beta>0$, the Lipschitz space $\operatorname{Lip}_{\beta}\left(R^{n}\right)$ is the space of functions $f$ such that

$$
\|f\|_{\operatorname{Lip}_{\beta}}=\sup _{\substack{x, y \in R^{n} \\ x \neq y}} \frac{|f(x)-f(y)|}{|x-y|^{\beta}}<\infty .
$$

In this paper, we will study some singular integral operators as follows (see [10]).

Definition 1 Fix $0 \leq \delta<n$. Let $T_{\delta}: S \rightarrow S^{\prime}$ be a linear operator such that $T_{\delta}$ is bounded on $L^{2}\left(R^{n}\right)$ and has a kernel $K$, that is, there exists a locally integrable function $K(x, y)$ on $R^{n} \times R^{n} \backslash\left\{(x, y) \in R^{n} \times R^{n}: x=y\right\}$ such that

$$
T_{\delta}(f)(x)=\int_{R^{n}} K(x, y) f(y) d y
$$


for every bounded and compactly supported function $f$, where for $K$ we have the following: there is a sequence of positive constant numbers $\left\{C_{k}\right\}$ such that for any $k \geq 1$,

$$
\int_{2|y-z|<|x-y|}(|K(x, y)-K(x, z)|+|K(y, x)-K(z, x)|) d x \leq C,
$$

and

$$
\begin{aligned}
& \left(\int_{2^{k}|z-y| \leq|x-y|<2^{k+1}|z-y|}(|K(x, y)-K(x, z)|+|K(y, x)-K(z, x)|)^{q} d y\right)^{1 / q} \\
& \quad \leq C_{k}\left(2^{k}|z-y|\right)^{-n / q^{\prime}+\delta}
\end{aligned}
$$

where $1<q^{\prime}<2$ and $1 / q+1 / q^{\prime}=1$. We write $T_{\delta} \in \operatorname{GSIO}(\delta)$.

Let $b$ be a locally integrable function on $R^{n}$. The commutator related to $T_{\delta}$ is defined by

$$
T_{\delta}^{b}(f)(x)=\int_{R^{n}}(b(x)-b(y)) K(x, y) f(y) d y .
$$

Remark (a) Note that the classical Calderón-Zygmund singular integral operator satisfies Definition 1 with $C_{j}=2^{-j \varepsilon}, \varepsilon>0$ and $\delta=0$ (see [12,13]).

(b) Also note that the fractional integral operator with rough kernel satisfies Definition 1 (see [2]), that is, for $0<\delta<n$, let $T_{\delta}$ be the fractional integral operator with rough kernel defined by (see [2])

$$
T_{\delta} f(x)=\int_{R^{n}} \frac{\Omega(x-y)}{|x-y|^{n-\delta}} f(y) d y,
$$

where $\Omega$ is homogeneous of degree zero on $R^{n}, \int_{S^{n-1}} \Omega\left(x^{\prime}\right) d \sigma\left(x^{\prime}\right)=0$ and $\Omega \in \operatorname{Lip}_{\varepsilon}\left(S^{n-1}\right)$ for some $0<\varepsilon \leq 1$, that is, there exists a constant $M>0$ such that for any $x, y \in S^{n-1}, \mid \Omega(x)-$ $\Omega(y)|\leq M| x-\left.y\right|^{\varepsilon}$. When $\Omega \equiv 1, T_{\delta}$ is the Riesz potential (fractional integral operator).

Definition 2 Let $\varphi$ be a positive, increasing function on $R^{+}$and there exists a constant $D>0$ such that

$$
\varphi(2 t) \leq D \varphi(t) \quad \text { for } t \geq 0
$$

Let $f$ be a locally integrable function on $R^{n}$. Set, for $0 \leq \eta<n$ and $1 \leq p<n / \eta$,

$$
\|f\|_{L^{p, \eta, \varphi},}=\sup _{x \in R^{n}, d>0}\left(\frac{1}{\varphi(d)^{1-p \eta / n}} \int_{Q(x, d)}|f(y)|^{p} d y\right)^{1 / p}
$$

where $Q(x, d)=\left\{y \in R^{n}:|x-y|<d\right\}$. The generalized fractional Morrey space is defined by

$$
L^{p, \eta, \varphi}\left(R^{n}\right)=\left\{f \in L_{\mathrm{loc}}^{1}\left(R^{n}\right):\|f\|_{L^{p, \eta, \varphi}}<\infty\right\} .
$$

We write $L^{p, \eta, \varphi}\left(R^{n}\right)=L^{p, \varphi}\left(R^{n}\right)$ if $\eta=0$, which is the generalized Morrey space. If $\varphi(d)=$ $d^{\eta}, \eta>0$, then $L^{p, \varphi}\left(R^{n}\right)=L^{p, \eta}\left(R^{n}\right)$, which is the classical Morrey spaces (see [7, 14]). If $\varphi(d)=1$, then $L^{p, \varphi}\left(R^{n}\right)=L^{p}\left(R^{n}\right)$, which is the Lebesgue spaces. 
As the Morrey space may be considered as an extension of the Lebesgue space, it is natural and important to study the boundedness of operator on Morrey spaces (see [3, 4, $6,15,16])$.

It is well known that commutators are of great interest in harmonic analysis, and they have been widely studied by many authors (see [2, 7]). In [7], Pérez and Trujillo-Gonzalez prove a sharp estimate for the commutator. The main purpose of this paper is to prove some sharp maximal inequalities for the commutator $T_{\delta}^{b}$. As an application, we obtain the $L^{p}$-norm inequality, and for Morrey and Triebel-Lizorkin spaces boundedness for the commutator.

\section{Theorems}

We shall prove the following theorems.

Theorem 1 Let there be a sequence $\left\{C_{k}\right\} \in l^{1}, 0<\beta<1, q^{\prime} \leq s<\infty$ and $b \in \operatorname{Lip}_{\beta}\left(R^{n}\right)$. Suppose $T_{\delta}$ is a bounded linear operator from $L^{p}\left(R^{n}\right)$ to $L^{r}\left(R^{n}\right)$ for any $p, r$ with $1<p<n / \delta$ and $1 / r=1 / p-\delta / n$, and that it has a kernel $K$ satisfying (1). Then there exists a constant $C>0$ such that, for any $f \in C_{0}^{\infty}\left(R^{n}\right)$ and $\tilde{x} \in R^{n}$,

$$
M^{\#}\left(T_{\delta}^{b}(f)\right)(\tilde{x}) \leq C\|b\|_{\operatorname{Lip}_{\beta}}\left(M_{\beta+\delta, s}(f)(\tilde{x})+M_{\beta, s}\left(T_{\delta}(f)\right)(\tilde{x})\right) .
$$

Theorem 2 Let there be a sequence $\left\{2^{k \beta} C_{k}\right\} \in l^{1}, 0<\beta<1, q^{\prime} \leq s<\infty$ and $b \in \operatorname{Lip}_{\beta}\left(R^{n}\right)$. Suppose $T_{\delta}$ is a bounded linear operator from $L^{p}\left(R^{n}\right)$ to $L^{r}\left(R^{n}\right)$ for any $p, r$ with $1<p<n / \delta$ and $1 / r=1 / p-\delta / n$, and that it has a kernel $K$ satisfying (1). Then there exists a constant $C>0$ such that, for any $f \in C_{0}^{\infty}\left(R^{n}\right)$ and $\tilde{x} \in R^{n}$,

$$
\operatorname{supinf}_{Q \ni \tilde{x} c} \frac{1}{|Q|^{1+\beta / n}} \int_{Q}\left|T_{\delta}^{b}(f)(x)-c\right| d x \leq C\|b\|_{\operatorname{Lip}_{\beta}}\left(M_{\delta, s}(f)(\tilde{x})+M_{s}\left(T_{\delta}(f)\right)(\tilde{x})\right) .
$$

Theorem 3 Let there be a sequence $\left\{k C_{k}\right\} \in l^{1}, q^{\prime} \leq s<\infty$ and $b \in B M O\left(R^{n}\right)$. Suppose $T_{\delta}$ is a bounded linear operator from $L^{p}\left(R^{n}\right)$ to $L^{r}\left(R^{n}\right)$ for any $p, r$ with $1<p<n / \delta$ and $1 / r=1 / p-\delta / n$, and that it has a kernel $K$ satisfying (1). Then there exists a constant $C>0$ such that, for any $f \in C_{0}^{\infty}\left(R^{n}\right)$ and $\tilde{x} \in R^{n}$,

$$
M^{\#}\left(T_{\delta}^{b}(f)\right)(\tilde{x}) \leq C\|b\|_{B M O}\left(M_{\delta, s}(f)(\tilde{x})+M_{s}\left(T_{\delta}(f)\right)(\tilde{x})\right)
$$

Theorem 4 Let there be a sequence $\left\{C_{k}\right\} \in l^{1}, 0<\beta<\min (1, n-\delta), q^{\prime}<p<n /(\beta+\delta)$, $1 / r=1 / p-(\beta+\delta) / n$ and $b \in \operatorname{Lip}_{\beta}\left(R^{n}\right)$. Suppose $T_{\delta}$ is a bounded linear operator from $L^{p}\left(R^{n}\right)$ to $L^{r}\left(R^{n}\right)$ and has a kernel $K$ satisfying (1). Then $T_{\delta}^{b}$ is bounded from $L^{p}\left(R^{n}\right)$ to $L^{r}\left(R^{n}\right)$.

Theorem 5 Let there be a sequence $\left\{C_{k}\right\} \in l^{1}, 0<D<2^{n}, 0<\beta<\min (1, n-\delta), q^{\prime}<p<$ $n /(\beta+\delta), 1 / r=1 / p-(\beta+\delta) / n$ and $b \in \operatorname{Lip}_{\beta}\left(R^{n}\right)$. Suppose $T_{\delta}$ is a bounded linear operator from $L^{p}\left(R^{n}\right)$ to $L^{r}\left(R^{n}\right)$ and has a kernel $K$ satisfying $(1)$. Then $T_{\delta}^{b}$ is bounded from $L^{p, \beta+\delta, \varphi}\left(R^{n}\right)$ to $L^{r, \varphi}\left(R^{n}\right)$.

Theorem 6 Let there be a sequence $\left\{2^{k \beta} C_{k}\right\} \in l^{1}, 0<\beta<1, q^{\prime}<p<n / \delta, 1 / r=1 / p-\delta / n$ and $b \in \operatorname{Lip}_{\beta}\left(R^{n}\right)$. Suppose $T_{\delta}$ is a bounded linear operator from $L^{p}\left(R^{n}\right)$ to $L^{r}\left(R^{n}\right)$ and has a kernel $K$ satisfying (1). Then $T_{\delta}^{b}$ is bounded from $L^{p}\left(R^{n}\right)$ to $\dot{F}_{r}^{\beta, \infty}\left(R^{n}\right)$. 
Theorem 7 Let there be a sequence $\left\{k C_{k}\right\} \in l^{1}, q^{\prime}<p<n / \delta, 1 / r=1 / p-\delta / n$ and $b \in$ $B M O\left(R^{n}\right)$. Suppose $T_{\delta}$ is a bounded linear operator from $L^{p}\left(R^{n}\right)$ to $L^{r}\left(R^{n}\right)$ and has a kernel $K$ satisfying (1). Then $T_{\delta}^{b}$ is bounded from $L^{p}\left(R^{n}\right)$ to $L^{r}\left(R^{n}\right)$.

Theorem 8 Let there be a sequence $\left\{k C_{k}\right\} \in l^{1}, 0<D<2^{n}, q^{\prime}<p<n / \delta, 1 / r=1 / p-\delta / n$ and $b \in B M O\left(R^{n}\right)$. Suppose $T_{\delta}$ is a bounded linear operator from $L^{p}\left(R^{n}\right)$ to $L^{r}\left(R^{n}\right)$ and has a kernel $K$ satisfying (1). Then $T_{\delta}^{b}$ is bounded from $L^{p, \delta, \varphi}\left(R^{n}\right)$ to $L^{r, \varphi}\left(R^{n}\right)$.

Corollary Let $T_{\delta} \in G S I O(\delta)$, that is, $T_{\delta}$ is the singular integral operator as Definition 1. Then Theorems 1-8 hold for $T_{\delta}^{b}$.

\section{Proofs of theorems}

To prove the theorems, we need the following lemma.

Lemma 1 (see [10]) Let $T_{\delta}$ be the singular integral operator as Definition 1. Then $T_{\delta}$ is bounded from $L^{p}\left(R^{n}\right)$ to $L^{r}\left(R^{n}\right)$ for $1<p<n / \delta$ and $1 / r=1 / p-\delta / n$.

Lemma 2 (see [17]) For $0<\beta<1$ and $1<p<\infty$, we have

$$
\|f\|_{\dot{F}_{p}^{\beta, \infty}} \approx\left\|\sup _{Q \ni} \frac{1}{|Q|^{1+\beta / n}} \int_{Q}\left|f(x)-f_{Q}\right| d x\right\|_{L^{p}} \approx\left\|\operatorname{supinf}_{Q \ni \cdot c} \frac{1}{|Q|^{1+\beta / n}} \int_{Q}|f(x)-c| d x\right\|_{L^{p}} .
$$

Lemma 3 (see [4]) Let $0<p<\infty$ and $w \in \bigcup_{1 \leq r<\infty} A_{r}$. Then, for any smooth function $f$ for which the left-hand side is finite,

$$
\int_{R^{n}} M(f)(x)^{p} w(x) d x \leq C \int_{R^{n}} M^{\#}(f)(x)^{p} w(x) d x .
$$

Lemma 4 (see $[2,16])$ Suppose that $0<\eta<n, 1<s<p<n / \eta$ and $1 / r=1 / p-\eta / n$. Then

$$
\left\|M_{\eta, s}(f)\right\|_{L^{r}} \leq C\|f\|_{L^{p}}
$$

Lemma 5 Let $1<p<\infty, 0<D<2^{n}$. Then, for any smooth function $f$ for which the left-hand side is finite,

$$
\|M(f)\|_{L^{p, \varphi}} \leq C\left\|M^{\#}(f)\right\|_{L^{p, \varphi}} .
$$

Proof For any cube $Q=Q\left(x_{0}, d\right)$ in $R^{n}$, we know $M\left(\chi_{Q}\right) \in A_{1}$ for any cube $Q=Q(x, d)$ by [18]. Noticing that $M\left(\chi_{Q}\right) \leq 1$ and $M\left(\chi_{Q}\right)(x) \leq d^{n} /\left(\left|x-x_{0}\right|-d\right)^{n}$ if $x \in Q^{c}$, by Lemma 3, we have, for $f \in L^{p, \varphi}\left(R^{n}\right)$,

$$
\begin{aligned}
\int_{Q} M(f)(x)^{p} d x & =\int_{R^{n}} M(f)(x)^{p} \chi_{Q}(x) d x \\
& \leq \int_{R^{n}} M(f)(x)^{p} M\left(\chi_{Q}\right)(x) d x \\
& \leq C \int_{R^{n}} M^{\#}(f)(x)^{p} M\left(\chi_{Q}\right)(x) d x
\end{aligned}
$$




$$
\begin{aligned}
= & C\left(\int_{Q} M^{\#}(f)(x)^{p} M\left(\chi_{Q}\right)(x) d x\right. \\
& \left.+\sum_{k=0}^{\infty} \int_{2^{k+1} Q \backslash 2^{k} Q} M^{\#}(f)(x)^{p} M\left(\chi_{Q}\right)(x) d x\right) \\
\leq & C\left(\int_{Q} M^{\#}(f)(x)^{p} d x+\sum_{k=0}^{\infty} \int_{2^{k+1} Q \backslash 2^{k} Q} M^{\#}(f)(x)^{p} \frac{|Q|}{\left|2^{k+1} Q\right|} d x\right) \\
\leq & C\left(\int_{Q} M^{\#}(f)(x)^{p} d x+\sum_{k=0}^{\infty} \int_{2^{k+1} Q} M^{\#}(f)(x)^{p} 2^{-k n} d y\right) \\
\leq & C\left\|M^{\#}(f)\right\|_{L^{p, \varphi}}^{p} \sum_{k=0}^{\infty} 2^{-k n} \varphi\left(2^{k+1} d\right) \leq C\left\|M^{\#}(f)\right\|_{L^{p, \varphi}}^{p} \sum_{k=0}^{\infty}\left(2^{-n} D\right)^{k} \varphi(d) \\
\leq & C\left\|M^{\#}(f)\right\|_{L^{p, \varphi}}^{p} \varphi(d),
\end{aligned}
$$

thus

$$
\left(\frac{1}{\varphi(d)} \int_{Q} M(f)(x)^{p} d x\right)^{1 / p} \leq C\left(\frac{1}{\varphi(d)} \int_{Q} M^{\#}(f)(x)^{p} d x\right)^{1 / p}
$$

and

$$
\|M(f)\|_{L^{p, \varphi}} \leq C\left\|M^{\#}(f)\right\|_{L^{p, \varphi}} .
$$

This finishes the proof.

Lemma 6 Let $T_{\delta}$ be a bounded linear operator from $L^{p}\left(R^{n}\right)$ to $L^{r}\left(R^{n}\right)$ for any $p, r$ with $1<p<n / \delta$ and $1 / r=1 / p-\delta / n, 0<D<2^{n}$. Then

$$
\left\|T_{\delta}(f)\right\|_{L^{r, \varphi}} \leq C\|f\|_{L^{p, \delta, \varphi}}
$$

Lemma 7 Let $0<D<2^{n}, 1 \leq s<p<n / \eta$ and $1 / r=1 / p-\eta / n$. Then

$$
\left\|M_{\eta, s}(f)\right\|_{L^{r, \varphi}} \leq C\|f\|_{L^{p, \eta, \varphi}}
$$

The proofs of two Lemmas are similar to that of Lemma 5 by Lemmas 1 and 4, we omit the details.

Proof of Theorem 1 It suffices to prove for $f \in C_{0}^{\infty}\left(R^{n}\right)$ and some constant $C_{0}$ that the following inequality holds:

$$
\frac{1}{|Q|} \int_{Q}\left|T_{\delta}^{b}(f)(x)-C_{0}\right| d x \leq C\|b\|_{\operatorname{Lip}_{\beta}}\left(M_{\beta+\delta, s}(f)(\tilde{x})+M_{\beta, s}\left(T_{\delta}(f)\right)(\tilde{x})\right) .
$$

Fix a cube $Q=Q\left(x_{0}, d\right)$ and $\tilde{x} \in Q$. Write, for $f_{1}=f \chi_{2 Q}$ and $f_{2}=f \chi_{(2 Q)}$,

$$
T_{\delta}^{b}(f)(x)=\left(b(x)-b_{2 Q}\right) T_{\delta}(f)(x)-T_{\delta}\left(\left(b-b_{2 Q}\right) f_{1}\right)(x)-T_{\delta}\left(\left(b-b_{2 Q}\right) f_{2}\right)(x) .
$$


Then

$$
\begin{aligned}
& \frac{1}{|Q|} \int_{Q}\left|T_{\delta}^{b}(f)(x)-T_{\delta}\left(\left(b_{2 Q}-b\right) f_{2}\right)\left(x_{0}\right)\right| d x \\
& \leq \frac{1}{|Q|} \int_{Q}\left|\left(b(x)-b_{2 Q}\right) T_{\delta}(f)(x)\right| d x+\frac{1}{|Q|} \int_{Q}\left|T_{\delta}\left(\left(b-b_{2 Q}\right) f_{1}\right)(x)\right| d x \\
& \quad+\frac{1}{|Q|} \int_{Q}\left|T_{\delta}\left(\left(b-b_{2 Q}\right) f_{2}\right)(x)-T_{\delta}\left(\left(b-b_{2 Q}\right) f_{2}\right)\left(x_{0}\right)\right| d x \\
& =I_{1}+I_{2}+I_{3} .
\end{aligned}
$$

For $I_{1}$, by Hölder's inequality, we obtain

$$
\begin{aligned}
I_{1} & \leq \frac{1}{|Q|} \sup _{x \in 2 Q}\left|b(x)-b_{2 Q}\right| \int_{Q}\left|T_{\delta}(f)(x)\right| d x \\
& \leq \frac{1}{|Q|} \sup _{x \in 2 Q}\left|b(x)-b_{2 Q}\right||Q|^{1-1 / s}\left(\int_{Q}\left|T_{\delta}(f)(x)\right|^{s} d x\right)^{1 / s} \\
& \leq C\|b\|_{\operatorname{Lip}_{\beta}}|Q|^{-1 / s}|2 Q|^{\beta / n}|Q|^{1 / s-\beta / n}\left(\frac{1}{|Q|^{1-s \beta / n}} \int_{Q}\left|T_{\delta}(f)(x)\right|^{s} d x\right)^{1 / s} \\
& \leq C\|b\|_{\operatorname{Lip}_{\beta}} M_{\beta, s}\left(T_{\delta}(f)\right)(\tilde{x}) .
\end{aligned}
$$

For $I_{2}$, choose $1<r<\infty$ such that $1 / r=1 / s-\delta / n$, by $\left(L^{s}, L^{r}\right)$-boundedness of $T_{\delta}$, we get

$$
\begin{aligned}
I_{2} & \leq\left(\frac{1}{|Q|} \int_{R^{n}}\left|T_{\delta}\left(\left(b-b_{2 Q}\right) f_{1}\right)(x)\right|^{r} d x\right)^{1 / r} \\
& \leq C|Q|^{-1 / r}\left(\int_{R^{n}}\left|\left(b(x)-b_{2 Q}\right) f_{1}(x)\right|^{s} d x\right)^{1 / s} \\
& \leq C|Q|^{-1 / r}\|b\|_{\operatorname{Lip}_{\beta}}|2 Q|^{\beta / n}|2 Q|^{1 / s-(\beta+\delta) / n}\left(\frac{1}{|2 Q|^{1-s(\beta+\delta) / n}} \int_{2 Q}|f(x)|^{s} d x\right)^{1 / s} \\
& \leq C\|b\|_{\operatorname{Lip}_{\beta}} M_{\beta+\delta, s}(f)(\tilde{x}) .
\end{aligned}
$$

For $I_{3}$, recalling that $s>q^{\prime}$, we have

$$
\begin{aligned}
I_{3} \leq & \frac{1}{|Q|} \int_{Q} \int_{(2 Q))^{c}}\left|b(y)-b_{2 Q}\right||f(y)|\left|K(x, y)-K\left(x_{0}, y\right)\right| d y d x \\
\leq & \frac{1}{|Q|} \int_{Q} \sum_{k=1}^{\infty} \int_{2^{k} d \leq\left|y-x_{0}\right| 2^{k+1} d}\left|K(x, y)-K\left(x_{0}, y\right)\right|\left|b(y)-b_{2^{k+1} Q}\right||f(y)| d y d x \\
& +\frac{1}{|Q|} \int_{Q} \sum_{k=1}^{\infty} \int_{2^{k} d \leq\left|y-x_{0}\right| 2^{k+1} d}\left|K(x, y)-K\left(x_{0}, y\right)\right|\left|b_{2^{k+1} Q}-b_{2 Q}\right||f(y)| d y d x \\
\leq & \frac{C}{|Q|} \int_{Q} \sum_{k=1}^{\infty}\left(\int_{2^{k} d \leq\left|y-x_{0}\right|<2^{k+1} d}\left|K(x, y)-K\left(x_{0}, y\right)\right|^{q} d y\right)^{1 / q} \\
& \times \sup _{y \in 2^{k+1} Q}\left|b(y)-b_{2^{k+1} Q}\right|\left(\int_{2^{k+1} Q}|f(y)|^{q^{\prime}} d y\right)^{1 / q^{\prime}} d x
\end{aligned}
$$




$$
\begin{aligned}
& +\frac{C}{|Q|} \int_{Q} \sum_{k=1}^{\infty}\left|b_{2^{k+1} Q}-b_{2 Q}\right|\left(\int_{2^{k} d \leq\left|y-x_{0}\right|<2^{k+1} d}\left|K(x, y)-K\left(x_{0}, y\right)\right|^{q} d y\right)^{1 / q} \\
& \times\left(\int_{2^{k+1} Q}|f(y)|^{q^{\prime}} d y\right)^{1 / q^{\prime}} d x \\
& \leq C \sum_{k=1}^{\infty} C_{k}\left(2^{k} d\right)^{-n / q^{\prime}+\delta}\left|2^{k+1} Q\right|^{\beta / n}\|b\|_{\operatorname{Lip}_{\beta}}\left|2^{k+1} Q\right|^{1 / q^{\prime}-1 / s}\left|2^{k+1} Q\right|^{1 / s-(\beta+\delta) / n} \\
& \times\left(\frac{1}{\left|2^{k+1} Q\right|^{1-s(\beta+\delta) / n}} \int_{2^{k+1} Q}|f(y)|^{s} d y\right)^{1 / s} \\
& +C \sum_{k=1}^{\infty}\|b\|_{\operatorname{Lip}_{\beta}}\left|2^{k} Q\right|^{\beta / n} C_{k}\left(2^{k} d\right)^{-n / q^{\prime}+\delta}\left|2^{k+1} Q\right|^{1 / q^{\prime}-1 / s}\left|2^{k+1} Q\right|^{1 / s-(\beta+\delta) / n} \\
& \times\left(\frac{1}{\left|2^{k+1} Q\right|^{1-s(\beta+\delta) / n}} \int_{2^{k+1} Q}|f(y)|^{s} d y\right)^{1 / s} \\
& \leq C\|b\|_{\operatorname{Lip}_{\beta}} M_{\beta+\delta, s}(f)(\tilde{x}) \sum_{k=1}^{\infty} C_{k} \leq C\|b\|_{\operatorname{Lip}_{\beta}} M_{\beta+\delta, s}(f)(\tilde{x}) .
\end{aligned}
$$

These complete the proof of Theorem 1.

Proof of Theorem 2 It suffices to prove for $f \in C_{0}^{\infty}\left(R^{n}\right)$ and some constant $C_{0}$ that the following inequality holds:

$$
\frac{1}{|Q|^{1+\beta / n}} \int_{Q}\left|T_{\delta}^{b}(f)(x)-C_{0}\right| d x \leq C\|b\|_{\operatorname{Lip}_{\beta}}\left(M_{\delta, s}(f)(\tilde{x})+M_{s}\left(T_{\delta}(f)\right)(\tilde{x})\right) .
$$

Fix a cube $Q=Q\left(x_{0}, d\right)$ and $\tilde{x} \in Q$. Write, for $f_{1}=f \chi_{2 Q}$ and $f_{2}=f \chi_{(2 Q)}$,

$$
T_{\delta}^{b}(f)(x)=\left(b(x)-b_{2 Q}\right) T_{\delta}(f)(x)-T_{\delta}\left(\left(b-b_{2 Q}\right) f_{1}\right)(x)-T_{\delta}\left(\left(b-b_{2 Q}\right) f_{2}\right)(x) .
$$

Then

$$
\begin{aligned}
& \frac{1}{|Q|^{1+\beta / n}} \int_{Q}\left|T_{\delta}^{b}(f)(x)-T_{\delta}\left(\left(b_{2 Q}-b\right) f_{2}\right)\left(x_{0}\right)\right| d x \\
& \leq \frac{1}{|Q|^{1+\beta / n}} \int_{Q}\left|\left(b(x)-b_{2 Q}\right) T_{\delta}(f)(x)\right| d x+\frac{1}{|Q|^{1+\beta / n}} \int_{Q}\left|T_{\delta}\left(\left(b-b_{2 Q}\right) f_{1}\right)(x)\right| d x \\
& \quad+\frac{1}{|Q|^{1+\beta / n}} \int_{Q}\left|T_{\delta}\left(\left(b-b_{2 Q}\right) f_{2}\right)(x)-T_{\delta}\left(\left(b-b_{2 Q}\right) f_{2}\right)\left(x_{0}\right)\right| d x \\
& =I I_{1}+I I_{2}+I I_{3} .
\end{aligned}
$$

By using the same argument as in the proof of Theorem 1, we get, for $1<r<\infty$ with $1 / r=1 / s-\delta / n$,

$$
\begin{aligned}
& I I_{1} \leq \frac{C}{|Q|^{1+\beta / n}} \sup _{x \in 2 Q}\left|b(x)-b_{2 Q}\right||Q|^{1-1 / s}\left(\int_{Q}\left|T_{\delta}(f)(x)\right|^{s} d x\right)^{1 / s} \\
& \leq C|Q|^{-1-\beta / n}\|b\|_{\operatorname{Lip}_{\beta}}|2 Q|^{\beta / n}|Q|^{1-1 / s}|Q|^{1 / s}\left(\frac{1}{|Q|} \int_{Q}\left|T_{\delta}(f)(x)\right|^{s} d x\right)^{1 / s} \\
& \leq C\|b\|_{\operatorname{Lip}_{\beta}} M_{s}\left(T_{\delta}(f)\right)(\tilde{x}),
\end{aligned}
$$




$$
\begin{aligned}
& I I_{2} \leq \frac{1}{|Q|^{1+\beta / n}}|Q|^{1-1 / r}\left(\int_{R^{n}}\left|T_{\delta}\left(\left(b-b_{2 Q}\right) f_{1}\right)(x)\right|^{r} d x\right)^{1 / r} \\
& \leq \frac{C}{|Q|^{1+\beta / n}}|Q|^{1-1 / r}\left(\int_{R^{n}}\left|\left(b(x)-b_{2 Q}\right) f_{1}(x)\right|^{s} d x\right)^{1 / s}
\end{aligned}
$$

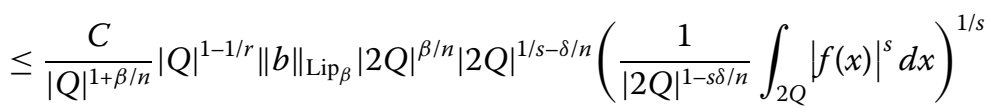

$$
\begin{aligned}
& \leq C\|b\|_{\operatorname{Lip}_{\beta}} M_{\delta, s}(f)(\tilde{x}) \text {, } \\
& I I_{3} \leq \frac{1}{|Q|^{1+\beta / n}} \int_{Q} \int_{(2 Q))^{c}}\left|b(y)-b_{2 Q}\right||f(y)|\left|K(x, y)-K\left(x_{0}, y\right)\right| d y d x \\
& \leq \frac{1}{|Q|^{1+\beta / n}} \int_{Q} \sum_{k=1}^{\infty} \int_{2^{k} d \leq\left|y-x_{0}\right|<2^{k+1} d}\left|K(x, y)-K\left(x_{0}, y\right)\right|\left|b(y)-b_{2^{k+1} Q}\right||f(y)| d y d x \\
& +\frac{1}{|Q|^{1+\beta / n}} \int_{Q} \sum_{k=1}^{\infty} \int_{2^{k} d \leq\left|y-x_{0}\right|<2^{k+1} d}\left|K(x, y)-K\left(x_{0}, y\right)\right|\left|b_{2^{k+1} Q}-b_{2 Q}\right||f(y)| d y d x \\
& \leq \frac{C}{|Q|^{1+\beta / n}} \int_{Q} \sum_{k=1}^{\infty}\left(\int_{2^{k} d \leq\left|y-x_{0}\right|<2^{k+1} d}\left|K(x, y)-K\left(x_{0}, y\right)\right|^{q} d y\right)^{1 / q} \\
& \times \sup _{y \in 2^{k+1} Q}\left|b(y)-b_{2^{k+1} Q}\right|\left(\int_{2^{k+1} Q}|f(y)|^{q^{\prime}} d y\right)^{1 / q^{\prime}} d x \\
& +\frac{C}{|Q|^{1+\beta / n}} \int_{Q} \sum_{k=1}^{\infty}\left|b_{2^{k+1} Q}-b_{2 Q}\right|\left(\int_{2^{k} d \leq\left|y-x_{0}\right|<2^{k+1} d}\left|K(x, y)-K\left(x_{0}, y\right)\right|^{q} d y\right)^{1 / q} \\
& \times\left(\int_{2^{k+1} Q}|f(y)|^{q^{\prime}} d y\right)^{1 / q^{\prime}} d x \\
& \leq C|Q|^{-\beta / n} \sum_{k=1}^{\infty} C_{k}\left(2^{k} d\right)^{-n / q^{\prime}+\delta}\left|2^{k+1} Q\right|^{\beta / n}\|b\|_{\operatorname{Lip}_{\beta}}\left|2^{k+1} Q\right|^{1 / q^{\prime}-\delta / n} \\
& \times\left(\frac{1}{\left|2^{k+1} Q\right|^{1-s \delta / n}} \int_{2^{k+1} Q}|f(y)|^{s} d y\right)^{1 / s} \\
& +C|Q|^{-\beta / n} \sum_{k=1}^{\infty}\|b\|_{\operatorname{Lip}_{\beta}}\left|2^{k} Q\right|^{\beta / n} C_{k}\left(2^{k} d\right)^{-n / q^{\prime}+\delta}\left|2^{k+1} Q\right|^{1 / q^{\prime}-\delta / n} \\
& \times\left(\frac{1}{\left|2^{k+1} Q\right|^{1-s \delta / n}} \int_{2^{k+1} Q}|f(x)|^{s} d x\right)^{1 / s} \\
& \leq C\|b\|_{\operatorname{Lip}_{\beta}} M_{\delta, s}(f)(\tilde{x}) \sum_{k=1}^{\infty} 2^{k \beta} C_{k} \\
& \leq C\|b\|_{\operatorname{Lip}_{\beta}} M_{\delta, s}(f)(\tilde{x}) .
\end{aligned}
$$

This completes the proof of Theorem 2 .

Proof of Theorem 3 It suffices to prove for $f \in C_{0}^{\infty}\left(R^{n}\right)$ and some constant $C_{0}$ that the following inequality holds:

$$
\frac{1}{|Q|} \int_{Q}\left|T_{\delta}^{b}(f)(x)-C_{0}\right| d x \leq C\|b\|_{B M O}\left(M_{\delta, s}(f)(\tilde{x})+M_{s}\left(T_{\delta}(f)\right)(\tilde{x})\right) .
$$


Fix a cube $Q=Q\left(x_{0}, d\right)$ and $\tilde{x} \in Q$. Write, for $f_{1}=f \chi_{2 Q}$ and $f_{2}=f \chi_{(2 Q)}$,

$$
T_{\delta}^{b}(f)(x)=\left(b(x)-b_{2 Q}\right) T_{\delta}(f)(x)-T_{\delta}\left(\left(b-b_{2 Q}\right) f_{1}\right)(x)-T_{\delta}\left(\left(b-b_{2 Q}\right) f_{2}\right)(x) .
$$

Then

$$
\begin{aligned}
& \frac{1}{|Q|} \int_{Q}\left|T_{\delta}^{b}(f)(x)-T_{\delta}\left(\left(b_{2 Q}-b\right) f_{2}\right)\left(x_{0}\right)\right| d x \\
& \leq \frac{1}{|Q|} \int_{Q}\left|\left(b(x)-b_{2 Q}\right) T_{\delta}(f)(x)\right| d x+\frac{1}{|Q|} \int_{Q}\left|T_{\delta}\left(\left(b-b_{2 Q}\right) f_{1}\right)(x)\right| d x \\
& \quad+\frac{1}{|Q|} \int_{Q}\left|T_{\delta}\left(\left(b-b_{2 Q}\right) f_{2}\right)(x)-T_{\delta}\left(\left(b-b_{2 Q}\right) f_{2}\right)\left(x_{0}\right)\right| d x \\
& =I I I_{1}+I I I_{2}+I I I_{3} .
\end{aligned}
$$

For $I I I_{1}$, by Hölder's inequality, we get

$$
\begin{aligned}
I I I_{1} & \leq\left(\frac{1}{|Q|} \int_{Q}\left|b(x)-b_{2 Q}\right|^{s^{\prime}} d x\right)^{1 / s^{\prime}}\left(\frac{1}{|Q|} \int_{Q}\left|T_{\delta}(f)(x)\right|^{s} d x\right)^{1 / s} \\
& \leq C\|b\|_{B M O} M_{s}\left(T_{\delta}(f)\right)(\tilde{x}) .
\end{aligned}
$$

For $I I I_{2}$, choose $1<p<s$ such that $1 / r=1 / p-\delta / n$, by Hölder's inequality and $\left(L^{p}, L^{r}\right)$ boundedness of $T_{\delta}$, we obtain

$$
\begin{aligned}
I I I_{2} \leq & \left(\frac{1}{|Q|} \int_{R^{n}}\left|T_{\delta}\left(\left(b-b_{2 Q}\right) f_{1}\right)(x)\right|^{r} d x\right)^{1 / r} \leq C|Q|^{-1 / r}\left(\int_{R^{n}}\left|\left(b-b_{2 Q}\right) f_{1}(x)\right|^{p} d x\right)^{1 / p} \\
\leq & C|Q|^{-1 / r}\left(\int_{2 Q}\left|b(x)-b_{2 Q}\right|^{s p /(s-p)} d x\right)^{(s-p) / s p}\left(\int_{2 Q}|f(x)|^{s} d x\right)^{1 / s} \\
\leq & C|Q|^{-1 / r}|2 Q|^{(s-p) / s p}\left(\frac{1}{|2 Q|} \int_{2 Q}\left|b(x)-b_{2 Q}\right|^{s p /(s-p)} d x\right)^{(s-p) / s p} \\
& \times|2 Q|^{1 / s-\delta / n}\left(\frac{1}{|2 Q|^{1-s \delta / n}} \int_{2 Q}|f(x)|^{s} d x\right)^{1 / s} \\
\leq & C\|b\|_{B M O}|Q|^{-1 / r+1 / p-\delta / n}\left(\frac{1}{|2 Q|^{1-s \delta / n}} \int_{2 Q}|f(x)|^{s} d x\right)^{1 / s} \\
\leq & C\|b\|_{B M O} M_{\delta, s}(f)(\tilde{x}) .
\end{aligned}
$$

For $I I I_{3}$, recalling that $s>q^{\prime}$, taking $1<p<\infty$ with $1 / p+1 / q+1 / s=1$, by Hölder's inequality, we obtain

$$
\begin{aligned}
I I I_{3} \leq & \frac{1}{|Q|} \int_{Q} \sum_{k=1}^{\infty} \int_{2^{k} d \leq\left|y-x_{0}\right|<2^{k+1} d}\left|K(x, y)-K\left(x_{0}, y\right)\right|\left|b(y)-b_{2 Q}\right||f(y)| d y d x \\
\leq & \frac{1}{|Q|} \int_{Q} \sum_{k=1}^{\infty}\left(\int_{2^{k} d \leq\left|y-x_{0}\right| 2^{k+1} d}\left|K(x, y)-K\left(x_{0}, y\right)\right|^{q} d y\right)^{1 / q} \\
& \times\left(\int_{2^{k+1} Q}\left|b(x)-b_{2 Q}\right|^{p} d x\right)^{1 / p}\left(\int_{2^{k+1} Q}|f(y)|^{s} d y\right)^{1 / s} d x
\end{aligned}
$$




$$
\begin{aligned}
\leq & C \sum_{k=1}^{\infty} C_{k}\left(2^{k} d\right)^{-n / q^{\prime}+\delta} k\left(2^{k} d\right)^{n / p}\|b\|_{B M O}\left(2^{k} d\right)^{n(1 / s-\delta / n)} \\
& \times\left(\frac{1}{\left|2^{k+1} Q\right|^{1-s \delta / n}} \int_{2^{k+1} Q}|f(y)|^{s} d y\right)^{1 / s} \\
\leq & C\|b\|_{B M O} \sum_{k=1}^{\infty} k C_{k}\left(2^{k} d\right)^{n(-1+1 / q+1 / p+1 / s)}\left(\frac{1}{\left|2^{k+1} Q\right|^{1-\delta \delta / n}} \int_{2^{k+1} Q}|f(y)|^{s} d y\right)^{1 / s} \\
\leq & C\|b\|_{B M O} M_{\delta, s}(f)(\tilde{x}) \sum_{k=1}^{\infty} k C_{k} \\
\leq & C\|b\|_{B M O} M_{\delta, s}(f)(\tilde{x}) .
\end{aligned}
$$

This completes the proof of Theorem 3.

Proof of Theorem 4 Choose $q^{\prime}<s<p$ in Theorem 1 and let $1 / t=1 / p-\delta / n$, then $1 / r=$ $1 / t-\beta / n$, thus we have, by Lemmas 1,3 , and 4 ,

$$
\begin{aligned}
\left\|T_{\delta}^{b}(f)\right\|_{L^{r}} & \leq\left\|M\left(T_{\delta}^{b}(f)\right)\right\|_{L^{r}} \leq C\left\|M^{\#}\left(T_{\delta}^{b}(f)\right)\right\|_{L^{r}} \\
& \leq C\|b\|_{\operatorname{Lip}_{\beta}}\left(\left\|M_{\beta, s}\left(T_{\delta}(f)\right)\right\|_{L^{r}}+\left\|M_{\beta+\delta, s}(f)\right\|_{L^{r}}\right) \\
& \leq C\|b\|_{\operatorname{Lip}_{\beta}}\left(\left\|T_{\delta}(f)\right\|_{L^{t}}+\|f\|_{L^{p}}\right) \\
& \leq C\|b\|_{\operatorname{Lip}_{\beta}}\|f\|_{L^{p}} .
\end{aligned}
$$

This completes the proof of Theorem 4 .

Proof of Theorem 5 Choose $q^{\prime}<s<p$ in Theorem 1 and let $1 / t=1 / p-\delta / n$, then $1 / r=$ $1 / t-\beta / n$, thus we have, by Lemmas $5-7$,

$$
\begin{aligned}
\left\|T_{\delta}^{b}(f)\right\|_{L^{r, \varphi}} & \leq\left\|M\left(T_{\delta}^{b}(f)\right)\right\|_{L^{r, \varphi}} \leq C\left\|M^{\#}\left(T_{\delta}^{b}(f)\right)\right\|_{L^{r, \varphi}} \\
& \leq C\|b\|_{\operatorname{Lip}_{\beta}}\left(\left\|M_{\beta, s}\left(T_{\delta}(f)\right)\right\|_{L^{r, \varphi}}+\left\|M_{\beta+\delta, s}(f)\right\|_{L^{r, \varphi}}\right) \\
& \leq C\|b\|_{\operatorname{Lip}_{\beta}}\left(\left\|T_{\delta}(f)\right\|_{L^{t, \beta, \varphi}}+\|f\|_{L^{p, \beta+\delta, \varphi}}\right) \\
& \leq C\|b\|_{\operatorname{Lip}_{\beta}}\|f\|_{L^{p, \beta+\delta, \varphi}} .
\end{aligned}
$$

This completes the proof of Theorem 5.

Proof Theorem 6 Choose $q^{\prime}<s<p$ in Theorem 2. By using Lemma 2, we obtain

$$
\begin{aligned}
\left\|T_{\delta}^{b}(f)\right\|_{\dot{F}_{r}^{\beta, \infty}} & \leq C\left\|\sup _{Q \ni \cdot} \frac{1}{|Q|^{1+\beta / n}} \int_{Q}\left|T_{\delta}^{b}(f)(x)-T_{\delta}\left(\left(b_{2 Q}-b\right) f_{2}\right)\left(x_{0}\right)\right| d x\right\|_{L^{r}} \\
& \leq C\|b\|_{\operatorname{Lip}_{\beta}}\left(\left\|M_{s}\left(T_{\delta}(f)\right)\right\|_{L^{r}}+\left\|M_{\delta, s}(f)\right\|_{L^{r}}\right) \\
& \leq C\|b\|_{\operatorname{Lip}_{\beta}}\left(\left\|T_{\delta}(f)\right\|_{L^{r}}+\|f\|_{L^{p}}\right) \\
& \leq C\|b\|_{\operatorname{Lip}_{\beta}}\|f\|_{L^{p}} .
\end{aligned}
$$

This completes the proof of Theorem 6 . 
Proof of Theorem 7 Choose $q^{\prime} \leq s<p$ in Theorem 3 and similar to the proof of Theorem 4, we have

$$
\begin{aligned}
\left\|T_{\delta}^{b}(f)\right\|_{L^{r}} & \leq\left\|M\left(T_{\delta}^{b}(f)\right)\right\|_{L^{r}} \leq C\left\|M^{\#}\left(T_{\delta}^{b}(f)\right)\right\|_{L^{r}} \\
& \leq C\|b\|_{B M O}\left(\left\|M_{s}\left(T_{\delta}(f)\right)\right\|_{L^{r}}+\left\|M_{\delta, s}(f)\right\|_{L^{r}}\right) \\
& \leq C\|b\|_{B M O}\left(\left\|T_{\delta}(f)\right\|_{L^{r}}+\|f\|_{L^{p}}\right) \\
& \leq C\|b\|_{B M O}\|f\|_{L^{p}} .
\end{aligned}
$$

This completes the proof of the theorem.

Proof of Theorem 8 Choose $q^{\prime} \leq s<p$ in Theorem 3 and similar to the proof of Theorem 5, we have

$$
\begin{aligned}
\left\|T_{\delta}^{b}(f)\right\|_{L^{r, \varphi}} & \leq\left\|M\left(T_{\delta}^{b}(f)\right)\right\|_{L^{r, \varphi}} \leq C\left\|M^{\#}\left(T_{\delta}^{b}(f)\right)\right\|_{L^{r, \varphi}} \\
& \leq C\|b\|_{B M O}\left(\left\|M_{s}\left(T_{\delta}(f)\right)\right\|_{L^{r, \varphi}}+\left\|M_{\delta, s}(f)\right\|_{L^{r, \varphi}}\right) \\
& \leq C\|b\|_{B M O}\left(\left\|T_{\delta}(f)\right\|_{L^{r, \varphi}}+\|f\|_{L^{p, \delta, \varphi}}\right) \\
& \leq C\|b\|_{B M O}\|f\|_{L^{p, \delta, \varphi}} .
\end{aligned}
$$

This completes the proof of the theorem.

\section{Competing interests}

The authors declare that they have no competing interests.

\section{Authors' contributions}

The authors completed the paper together. They also read and approved the final manuscript.

\section{Acknowledgements}

Project supported by Hunan Provincial Natural Science Foundation of China (12JJ6003) and Scientific Research Fund of Hunan Provincial Education Departments (12K017).

Received: 9 March 2014 Accepted: 25 April 2014 Published: 23 May 2014

\section{References}

1. Coifman, R, Rochberg, R, Weiss, G: Factorization theorems for Hardy spaces in several variables. Ann. Math. 103, 611-635 (1976)

2. Chanillo, S: A note on commutators. Indiana Univ. Math. J. 31, 7-16 (1982)

3. Di Fazio, G, Ragusa, MA: Commutators and Morrey spaces. Boll. Unione Mat. Ital., A (7) 5, 323-332 (1991)

4. Di Fazio, G, Ragusa, MA: Interior estimates in Morrey spaces for strong solutions to nondivergence form equations with discontinuous coefficients. J. Funct. Anal. 112, 241-256 (1993)

5. Janson, S: Mean oscillation and commutators of singular integral operators. Ark. Mat. 16, 263-270 (1978)

6. Liu, LZ: Interior estimates in Morrey spaces for solutions of elliptic equations and weighted boundedness for commutators of singular integral operators. Acta Math. Sci. 25(1), 89-94 (2005)

7. Pérez, C, Trujillo-Gonzalez, R: Sharp weighted estimates for multilinear commutators. J. Lond. Math. Soc. 65, 672-692 (2002)

8. Chen, WG: Besov estimates for a class of multilinear singular integrals. Acta Math. Sin. 16, 613-626 (2000)

9. Paluszynski, M: Characterization of the Besov spaces via the commutator operator of Coifman, Rochberg and Weiss. Indiana Univ. Math. J. 44, 1-17 (1995)

10. Chang, DC, Li, JF, Xiao, J: Weighted scale estimates for Calderón-Zygmund type operators. Contemp. Math. 446, 61-70 (2007)

11. Lin, Y: Sharp maximal function estimates for Calderón-Zygmund type operators and commutators. Acta Math. Sci. Ser. A Chin. Ed. 31, 206-215 (2011)

12. Garcia-Cuerva, J, Rubio de Francia, JL: Weighted Norm Inequalities and Related Topics. North-Holland Mathematics Studies, vol. 16. North-Holland, Amsterdam (1985)

13. Stein, EM: Harmonic Analysis: Real Variable Methods, Orthogonality and Oscillatory Integrals. Princeton University Press, Princeton (1993) 
14. Peetre, J: On the theory of $L^{p, \lambda}$-spaces. J. Funct. Anal. 4, 71-87 (1969)

15. Chiarenza, F, Frasca, M: Morrey spaces and Hardy-Littlewood maximal function. Rend. Mat. 7, 273-279 (1987)

16. Mizuhara, T: Boundedness of some classical operators on generalized Morrey spaces. In: Harmonic Analysis. ICM-90 Satellite Conference Proceedings (Sendai, 1990), pp. 183-189 (1991)

17. Muckenhoupt, B, Wheeden, RL: Weighted norm inequalities for fractional integral. Trans. Am. Math. Soc. 192, 261-274 (1974)

18. Coifman, R, Rochberg, R: Another characterization of BMO. Proc. Am. Math. Soc. 79, 249-254 (1980)

10.1186/1029-242X-2014-211

Cite this article as: Gu and Cai: Sharp maximal function inequalities and boundedness for commutators related to generalized fractional singular integral operators. Journal of Inequalities and Applications 2014, 2014:211

\section{Submit your manuscript to a SpringerOpen ${ }^{\circ}$} journal and benefit from:

- Convenient online submission

Rigorous peer review

- Immediate publication on acceptance

Open access: articles freely available online

- High visibility within the field

- Retaining the copyright to your article 